\title{
Applications of nanobodies in plant science and biotechnology
}

\author{
Wenyi Wang ${ }^{1,2,3} \cdot$ Jumao Yuan $^{2,3} \cdot$ Changan Jiang $^{2,3}$
}

Received: 28 June 2020 / Accepted: 5 October 2020 / Published online: 10 October 2020

(c) Springer Nature B.V. 2020

\begin{abstract}
Key message Present review summarizes the current applications of nanobodies in plant science and biotechnology, including plant expression of nanobodies, plant biotechnological applications, nanobody-based immunodetection, and nanobody-mediated resistance against plant pathogens.

Abstract Nanobodies (Nbs) are variable domains of heavy chain-only antibodies (HCAbs) isolated from camelids. In spite of their single domain structure, nanobodies display many unique features, such as small size, high stability, and cryptic epitopes accessibility, which make them ideal for sophisticated applications in plants and animals. In this review, we summarize the current applications of nanobodies in plant science and biotechnology, focusing on nanobody expression in plants, plant biotechnological applications, determination of plant toxins and pathogens, and nanobody-mediated resistance against plant pathogens. Prospects and challenges of nanobody applications in plants are also discussed.
\end{abstract}

Keywords Nanobody $\cdot \mathrm{V}_{\mathrm{H}} \mathrm{H} \cdot$ Single-domain antibody $\cdot$ Plant biotechnology $\cdot$ Nanobiotechnology

\begin{tabular}{|c|c|}
\hline \multicolumn{2}{|c|}{ Abbreviations } \\
\hline AID & Auxin-inducible degron \\
\hline CDR & Complementary determining region \\
\hline $\mathrm{CH}$ & Constant heavy-chain domain \\
\hline CL & Constant light-chain domain \\
\hline ER & Endoplasmic reticulum \\
\hline FR & Framework region \\
\hline GM & Genetically modified \\
\hline HCAbs & Heavy chain-only antibodies \\
\hline $\operatorname{Igs}$ & Immunoglobulins \\
\hline mAbs & Monoclonal antibodies \\
\hline $\mathrm{MV}_{\mathrm{H}} \mathrm{H}-\mathrm{IgA}$ & Monomeric $\mathrm{V}_{\mathrm{H}} \mathrm{H}-\operatorname{Ig} \mathrm{A}$ \\
\hline $\mathrm{Nbs}$ & Nanobodies \\
\hline POIs & Proteins of interest \\
\hline $\mathrm{SCF}$ & SKP1-CUL1-F-box \\
\hline
\end{tabular}

Wenyi Wang

wangwenyihaida@163.com

1 Institute for Medical Biology and Hubei Provincial Key Laboratory for Protection and Application of Special Plants in Wuling Area of China, College of Life Sciences, SouthCentral University for Nationalities, Wuhan, Hubei, China

2 Precision Medicine R\&D Center, Zhuhai Institute of Advanced Technology, Chinese Academy of Sciences, Zhuhai, Guangdong Province, China

3 Institute of Biomedicine and Biotechnology, Shenzhen Institutes of Advanced Technology, Chinese Academy of Sciences, Shenzhen, Guangdong Province, China

$\begin{array}{ll}\text { scFvs } & \text { Single-chain Fv fragments } \\ \text { sdAbs } & \text { Single-domain antibody } \\ \text { SELs } & \text { Size exclusion limits } \\ \text { SIgA } & \text { Secretory } \operatorname{IgA} \\ \text { SV }_{\mathrm{H}} \mathrm{H}-\operatorname{Ig} \mathrm{A} & \text { Secretory } \mathrm{V}_{\mathrm{H}} \mathrm{H}-\mathrm{Ig} \mathrm{A} \\ \mathrm{UPS} & \text { Ubiquitin-proteasome system } \\ \text { VH } & \text { Variable heavy-chain domain } \\ \mathrm{V}_{\mathrm{H}} \mathrm{H} & \text { Variable domains of the heavy chain of } \\ & \text { HCAbs } \\ \text { VL } & \text { Variable light-chain domain }\end{array}$

\section{Introduction}

Heavy chain-only antibodies (HCAbs) ( $95 \mathrm{kDa})$ are immunoglobulins naturally devoid of light chains in the serum of camelid (llamas, camels, alpacas, guanacos, and dromedaries), and the recombinant variable domains of the heavy chain of HCAbs $\left(\mathrm{V}_{\mathrm{H}} \mathrm{H}\right)$ are called nanobodies $(\mathrm{Nbs})$ or single-domain antibody (sdAbs) (Muyldermans 2013). Nanobodies are the smallest currently known antigen-binding proteins ( $4 \mathrm{~nm}$ in length and $2.5 \mathrm{~nm}$ in diameter). With molecular weights of $12-15 \mathrm{kDa}$, they are approximate onetenth the size of conventional mammalian Abs $(\sim 150 \mathrm{kDa})$ (Chakravarty et al. 2014; Jovcevska and Muyldermans 2020) (Fig. 1). Despite their small sizes, nanobodies bind their epitopes with high specificity and strong affinity. The 


\section{Conventional antibody $-150 \mathrm{kDa}$}

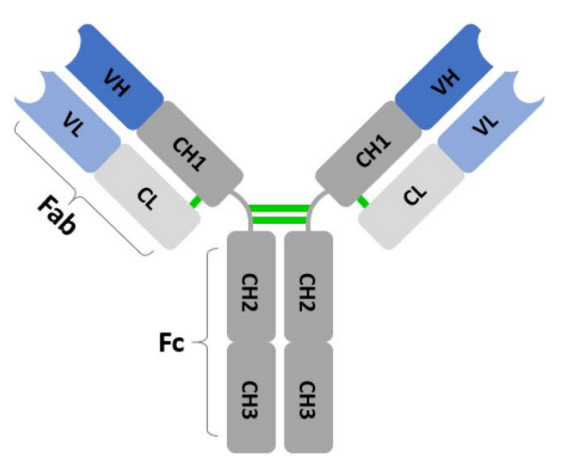

Fig. 1 Schematic representation of conventional antibodies and camelid heavy chain-only antibodies (HCAbs) derived nanobodies. Conventional antibodies are composed of two identical heavy chains and two identical light chains, with heavy chains containing three constant domains (CH1-3, dark gray) and one variable domain (VH, dark blue), and light chains containing one constant domain (CL, light gray) as well as one variable domain (VL, light blue). Variable regions of each chains are CDRs responsible for antigen recognition. Compared with conventional antibodies, camelid HCAbs

dissociation constants of nanobodies can reach nanomolar even picomolar range, which are comparable to those of conventional Abs (Ingram et al. 2018; Liu et al. 2018).

Nanobodies show several advantages over conventional monoclonal antibodies (mAbs). First, nanobodies present high solubility and stability even under stringent conditions. With high sequence homology to VH domains of human immunoglobulins (Igs), nanobodies also consist of four conserved framework regions (FR1-4) which maintain the core structure and three complementary determining regions (CDR1-3) that are responsible for specific recognition of antigens (Muyldermans et al. 2009). Compared with VH domains of IgGs, hydrophobic amino acids maintaining VH/VL interaction within FR are mutated to hydrophilic amino acids, which improve the solubility and stability of nanobodies (Schumacher et al. 2018). Nanobodies can be concentrated to a concentration as high as $1-10 \mathrm{mg} / \mathrm{mL}$ in standard phosphate or Tris buffers (Muyldermans 2013). As is known that conventional mAbs are vulnerable to harsh environmental conditions as a result of their multimeric structure (Schumacher et al. 2018), while monomeric nanobodies exhibit excellent tolerance to extreme temperature $\left(60-80{ }^{\circ} \mathrm{C}\right.$, even $\left.90{ }^{\circ} \mathrm{C}\right)$ and $\mathrm{pH}(\mathrm{pH} 3.0-9.0)$ conditions, which probably result from their efficient refolding capability after denaturation without compromising their antigen-binding capacity (De Vos et al. 2013; Harmsen and De Haard 2007). Furthermore, apart from one conserved disulfide bond, an extra interloop disulfide bond may exist for many camel $\mathrm{V}_{\mathrm{H}} \mathrm{Hs}$, and additional introduced disulfide bond has been reported to stabilize nanobodies and increase
Camelid HCAb

$\sim 95 \mathrm{kDa}$

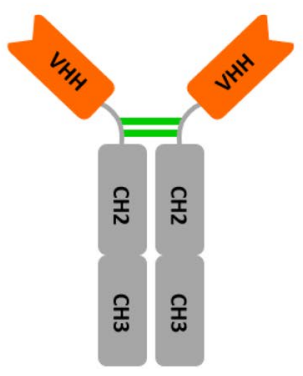

Nanobody

12-15 kDa lack the light chains and the $\mathrm{CH} 1$ domain of the heavy chains, and recognizes antigens through a single variable domain $\mathrm{V}_{\mathrm{H}} \mathrm{H}$ (orange), which is also called nanobody. Interchain disulfide bonds are labeled and colored in green. $\mathrm{CH}$ constant heavy-chain domain, $\mathrm{VH}$ variable heavy-chain domain, $C L$ constant light-chain domain, $V L$ variable light-chain domain, $C D R$ complementary determining region, $H C A b s$ heavy chain-only antibodies, $V_{H} H$ variable domains of the heavy chain of HCAbs

their resistance to pepsin or chymotrypsin degradation, suggesting the possibility of oral administration of such nanobodies (Hussack et al. 2011; Ingram et al. 2018). Second, nanobodies are amenable to genetic engineering for their monomeric structure. Different proteins can be fused with nanobodies conveniently to achieve specificity, facilitating multifunctional molecular operation. Unlike most mAbs which can only be efficiently manufactured in mammalian cells, nanobodies can be produced in bacteria and yeast due to their lack of $\mathrm{Fc}$ fragment with $\mathrm{N}$-linked oligosaccharide. Large-scale and cost-effective microbial production systems have been developed for nanobodies (Liu and Huang 2018). Since certain disulfide bonds in nanobodies contribute to their protein stability, it is preferred to produce nanobodies in the oxidizing periplasmic space of Escherichia coli or in the engineered $E$. coli strain with less reducing cytosol which is conducive for proper disulfide bonds formation (Liu and Huang 2018; Manta et al. 2019). Eukaryotic microbial hosts such as yeast enable efficient formation of the disulfide bond and are suitable to produce soluble functional nanobodies, but wrong $\mathrm{N}$-glycosylation occasionally happened (Liu and Huang 2018). Plants are a preferable expression system for nanobodies owing to reasons that will be discussed below. Third, nanobodies can recognize cryptic concave epitopes, such as enzyme active sites, which are inaccessible for conventional mAbs. This unique capability is probably conferred by their convex paratope and also their flexible long CDR3 loop, which implies that nanobodies can be used as enzyme inhibitors (De Genst et al. 2006; Desmyter et al. 1996; Jobling et al. 2002). Besides, nanobodies are superior 
to conventional mAbs because of their low immunogenicity, deep tissue penetration, and fast blood clearance, and the short half-life of nanobodies conferred by their small size can be prolonged by Fc fusion (Hutt et al. 2012), all of which make nanobodies star proteins with a wide range of applications in mammals as diagnostic or therapeutic drugs (Salvador et al. 2019; Wang et al. 2016b).

The prevalent technique for nanobody generation is screening libraries constructed from immunized camelids by phage display. Alternatively, nanobodies can be isolated from naïve libraries constructed from non-immunized camelids. Generally, naïve libraries provide nanobodies with high diversity but limited specificity and affinity, while on the contrary, immune libraries provide nanobodies with high affinity but limited diversity due to the affinity maturation process by somatic hypermutation during animal immunization (Moutel et al. 2016). In the last few years, synthetic libraries have also been developed. Synthetic libraries are constructed artificially by randomizing the CDRs using selected nanobody scaffold and thus are suitable for screening nanobodies against any antigen, including toxic and lowimmunogenic antigens that are challenging for conventional animal immunization approach (Moutel et al. 2016; Yan et al. 2014). Unlike the immune libraries which require the construction of a new library for each antigen, the synthetic libraries are suitable for screening nanobodies against different antigens (Liu et al. 2018). However, the nanobodies isolated from synthetic libraries often show lower affinity, presumably due to lack of in vivo antibody maturation process, but this drawback could be ameliorated by increasing the size and diversity of the synthetic libraries, as well as adopting an optimized screening method. Recently, a protocol of nanobody screening using synthetic library has been published, through which one can generate synthetic nanobodies efficiently against most antigens within three weeks (Zimmermann et al. 2020).

The unique features of nanobodies have raised significant interest among plant biologists. Research using nanobodies in plants has been increasing rapidly in recent years. In this review, we discuss progress of nanobody applications in plants, including plant-based nanobody expression, nanobody-mediated functional modulation, toxin and pathogen detection, as well as nanobody technologies for plant pathogen resistance (Fig. 2).

\section{Nanobody expression in plants}

Recombinant proteins expressed in mammalian cells are well folded with accurate post-translational modification, thus to date, most diagnostic and therapeutic antibodies are still produced with mammalian cells. However, the mammalian-based expression system is also associated with disadvantages of high cost and risk of pathogen contamination (Liu and Huang 2018). As mentioned above, E. coli is one of the most popular host for nanobody expression, yet risk still exists as a result of endotoxin contamination and the formation of inclusion bodies (Arbabi-Ghahroudi et al. 2005).

Plants are considered as premier alternative expression hosts for nanobodies owing to their easy transformation and cultivation, low scale-up costs, low risk of pathogen contamination, efficient post-translational modifications, as well as antibodies correct assembly, and the production of diagnostic and therapeutic proteins in plants is commonly described as "molecular farming" (Orzaez et al. 2009; Tschofen et al. 2016). Another reason why nanobodies are suitable to be produced and function in the reducing cytoplasm of plant cells, which is not conducive to disulfide bond formation, is that the folding and stability of nanobodies are less dependent on the formation of disulfide bonds (Ingram et al. 2018). Nanobody expression in plant leaf facilitates scaling-up, which basically depends on the expansion of the planting area with increased water and fertilizer supply, and higher production can be obtained by multiple harvests per year. Tobacco is the most popular species thanks to the high amount of leaf biomass and easy genetic manipulations, including Agrobacterium-mediated stable transformation of tobacco and also Agrobacterium infiltration or viral vector-mediated transient expression, which enables rapid protein production in a few weeks (Whaley et al. 2011). The nanobody yields in tobacco can be as high as $1.7 \%$ of total soluble proteins (Conrad et al. 2011; Rajabi-Memari et al. 2006), and when targeted to the apoplast, the yield can even reach to $30 \%$ of total leaf protein (Teh and Kavanagh 2010). However, plant leaf expression demands for immediate protein extraction after leaf harvesting due to their short shelf life, thus causing inconvenience of leaf storage, which can be overcome by applying plant seed-based expression platforms (Xu et al. 2012). Antibody production in plant seeds (Arabidopsis, rice, soybean, wheat, maize, etc.) enables long-term storage while maintaining immunogenicity, even at ambient temperature with high protein concentrations, and their transportation can be carried out without cold chain maintenance, thus greatly reducing costs (Boothe et al. 2010; $\mathrm{Xu}$ et al. 2012). Besides, it is convenient and cost-effective to extract and purify antibodies from plant seeds (Abe et al. 2014; Virdi et al. 2015). It should be noted that a seed-specific promoter (e.g., the $\beta$-phaseolin promoter) instead of the cauliflower mosaic virus (CaMV) $35 \mathrm{~S}$ promoter should be used to drive transgene expression in plant seeds (Van Droogenbroeck et al. 2007).

Plant-based nanobody production can be further improved by various methods including usage of Agrobacterium infiltration-mediated transient expression system (Vezina et al. 2009), transformation strategies (Palaci et al. 


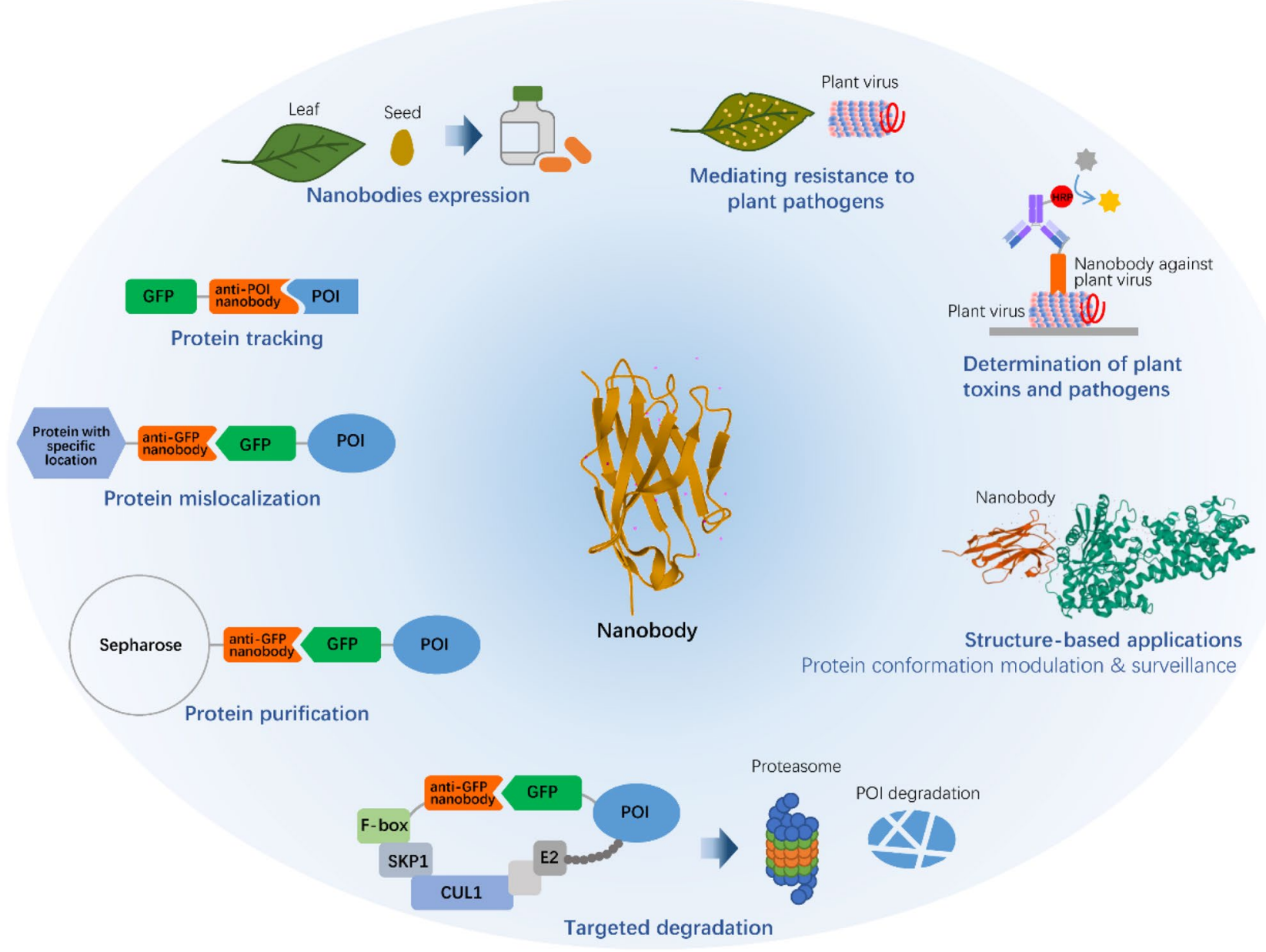

Fig. 2 Applications of nanobodies in plant science and biotechnology. Camelid derived nanobody is exemplified by the structure of the anti-GFP nanobody (PDB code, 3OGO), and the structure of the

2019), codon-optimization (Streatfield 2007), RNA interference technology (Abe et al. 2014), subcellular targeting (chloroplast, apoplast, etc.) (Alvarez et al. 2010; Barrera et al. 2015; Jin and Daniell 2015; Lentz et al. 2012; Mirzaee et al. 2018; Soleimanizadeh et al. 2018; Winichayakul et al. 2009), translational fusion with Fc domain or other stabilizing partners (De Buck et al. 2013; Lentz et al. 2012; Soleimanizadeh et al. 2018; Winichayakul et al. 2009), and suppression of post-transcriptional gene silencing (Alvarez et al. 2008). It is worth noting that N-glycosylation in plants differs from that in mammals, and this might lead to dysfunctional retention of the nanobody-Fc fusion proteins in the endoplasmic reticulum (ER) of plants (Liu and Huang 2018). Besides, allergic reactions might be caused by plant specific $\alpha(1,3)$-fucose and $\beta(1,2)$-xylose glycan residues when administrated to humans, thus ER retention sequence (H/KDEL) can be applied to retain the glycoproteins in the complex of EDS1 and its specific nanobody (PDB code, 6I8G) is presented in "Structure-based applications"

ER for reducing plant-specific glycosylation (Daniell et al. 2009; Park et al. 2020). Apart from that, various glycolengineering approaches have been developed to control the glycosylation of plant-produced recombinant proteins (Chen et al. 2005; Strasser et al. 2014).

Since nanobodies retain high stability even in harsh environmental conditions, they can be produced in edible plant tissues (roots, seeds, tubers, leaves, fruits, etc.) and orally administered to induce passive immunization of mammals, especially farm animals. In this process, the plant matrix itself provides protection for the recombinant protein from gastric digestion probably through outcompeting proteases, and the protective effect is enhanced when the recombinant protein is expressed in storage organelles (e.g., vacuoles, or oil bodies) because of endomembrane protection (Topp et al. 2016; Zimmermann et al. 2009). The conventional Igs used in passive immunization include $\operatorname{IgG}$ for intravenous injection and $\operatorname{Ig} \mathrm{A}$ 
for intestinal tract. Their production involves heavy chain and light chain expression and correct assembly, and the genes encoding J-chain and secretory component are also required for secretory $\operatorname{Ig} \mathrm{A}(\mathrm{SIgA})$, which is very complex and challenging (De Greve et al. 2020). Nanobodies can be grafted on to the Fc domain of Immunoglobulin ( $\operatorname{IgG}$ or $\operatorname{Ig} \mathrm{A}$ ) at the hinge region to make monomeric nanobody- $\mathrm{Fc}$ antibodies, which are easy to manufacture because they are encoded by a single gene (De Greve et al. 2020). Fc domain fusion extends their circulatory half-life to avoid rapid clearance from host (Hutt et al. 2012), and is proved to be capable of boosting the nanobody-Fc protein accumulation levels in Arabidopsis seeds (De Buck et al. 2013). Additionally, Fc domain fusion results in multiple valences, which is required for antibodies to block bacterial colonization through agglutination, and Fc domain also confers effector functions to the fused antibodies (De Greve et al. 2020; Roche et al. 2015).

Up to now, there have been a host of applications for plant production of nanobodies. In order to protect against enterotoxigenic Escherichia coli (ETEC) caused piglet postweaning diarrhea (PWD), a gastrointestinal infection occurring worldwide, Virdi et al. (2013) fused nanobodies against ETEC with the $\mathrm{Fc}$ region of $\mathrm{IgG}$ or IgA to promote ETEC agglutination and inhibited bacterial binding to porcine gut villous enterocytes. The chimeric antibodies were successfully expressed in Arabidopsis thaliana seeds with production levels as high as $3 \%$ of the seed weight, and the antibody-containing seeds were oral administrated to weaned piglets. The authors found that piglets fed with simplified secretory $\mathrm{V}_{\mathrm{H}} \mathrm{H}-\mathrm{IgA}\left(\mathrm{SV}_{\mathrm{H}} \mathrm{H}-\mathrm{Ig} \mathrm{A}\right)$ were protected from pathogens and gained more weight. Further analysis showed a decline in bacteria shedding and lower immune responses, suggesting reduced ETEC exposure to the weaned piglets. Since the plant expression of $\mathrm{SV}_{\mathrm{H}} \mathrm{H}-\mathrm{IgA}$ still involves introduction of three genes (including $\mathrm{V}_{\mathrm{H}} \mathrm{H}-\mathrm{IgA}$, J-chain, and secretory component) which is complex and timeconsuming, the authors further investigated the simplified single-gene-encoded monomeric $\mathrm{V}_{\mathrm{H}} \mathrm{H}-\mathrm{IgA}\left(\mathrm{MV}_{\mathrm{H}} \mathrm{H}-\mathrm{IgA}\right)$ and found it to be effective as well in preventing ETEC infection (Virdi et al. 2019). Similar strategies have been successfully adopted to express nanobodies directed against pathogens in plant tissues to protect livestock and poultry from enteric diseases (Harmsen et al. 2005; Saberianfar et al. 2019; Vanmarsenille et al. 2018). Besides, several nanobodies against toxins (Barrera et al. 2015; Richard et al. 2013), proteins involved in tumorigenesis (Ismaili et al. 2007; Mirzaee et al. 2018; Modarresi et al. 2018; Park et al. 2020; Rajabi-Memari et al. 2006; Soleimanizadeh et al. 2018), or key regulators of inflammatory diseases (Abe et al. 2014; Conrad et al. 2011; Giersberg et al. 2010; Winichayakul et al. 2009) have been produced successfully in plants, and are proved to be effective.

\section{Applications of nanobodies in plant biotechnology}

As outlined above, nanobodies are small-sized, highly stable and specific single-domain intrabodies that can be expressed within cells and recognize their antigens. Unlike conventional mAbs, nanobodies have no interchain disulfide bonds and give a low reliance on intrachain disulfide bonds for their stability and specificity, thus enabling nanobodies to maintain their antigen-binding activity and function well in the reducing cytosol environment. Additionally, the nanobodies are featured with the capability of recognizing cryptic epitopes of antigens as well as the relative ease of screening, expression, and fusion with other proteins, all of which make nanobodies effective tools for intracellular protein manipulation and analysis (Ingram et al. 2018; Jobling et al. 2002). Here we will discuss nanobody-based new technologies in plant researches.

\section{Protein tracking}

Conventional antibodies are widely used for protein visualization through immunostaining in fixed cells, but their application in living cells is constricted by their inefficient folding and chains assembly, which can be overcome by using nanobodies. To facilitate in vivo real-time tracing of proteins of interest (POIs), a fluorescent protein is commonly translational fused with nanobodies against these POIs, resulting in fluorescent, antigen-binding nanobodies which are called "chromobodies" (Rothbauer et al. 2006). Nanobody-based tracing technology enables tracking of the endogenous proteins of plants, which cannot be achieved by traditional GFP fusion tracing method, thus allowing for visualizing endogenous cellular activities with minimal interference. To visualize in vivo actin dynamics in leaf cells of tobacco, Rocchetti et al. (2014) adopted actin chromobodies to successfully label the actin cytoskeleton with less disturbance on Golgi body movement manner than the conventional labeling probes.

\section{Protein mislocalization and protein purification}

Nanobodies can be used to re-targeting POIs to artificially alter their distribution pattern, which is a knock-down strategy for protein functions evaluation. Nanobodies against GFP, originally isolated by Rothbauer et al. (2008), can be anchored to distinct cellular compartments or structures for the ectopical recruitment of GFP-tagged POIs, and thus serve as intracellular "nanotraps" (Borg et al. 2015; Rothbauer et al. 2008). In the model plant Nicotiana benthamiana, chromobodies were demonstrated to be effective 
in cytoplasm trapping and protein function alteration of GFP-tagged POIs, which are originally localized in endomembrane compartment, nucleus, plastid, or mitochondria (Schornack et al. 2009). However, chromobodies alone has been used to trace GFP-tagged POIs instead of altering their subcellular localization in HeLa cells (Rothbauer et al. 2006), the difference might be caused by the ratio of chromobodies versus target GFP protein between different species. Further, to artificially recruit GFP-tagged proteins to a specific location, the anti-GFP nanobodies can be covalently linked to a protein with a specific location within the cell (Rothbauer et al. 2008). To probe into the mechanism of sorting and degradation of soluble proteins in the vacuole, an nanobody-epitope interaction-based approach was adopted to track and lock down vacuolar sorting receptors (VSRs) into a specific compartment in mesophyll protoplasts of Nicotiana tabacum, thereby demonstrating that VSRs bound vacuole-targeted proteins in the ER and Golgi, and the recycled VSRs accomplished the reloading in the cis-Golgi (Fruholz et al. 2018; Kunzl et al. 2016). Other than manipulating individual protein, nanobodies are also capable of delocalizing multiple proteins simultaneously. Taking advantage of anti-GFP nanobodies binding to GFP and also its derivatives, Winkler et al. (2020) used mitochondria targeting anti-GFP nanobodies to delocalized the GFPtagged functional TML protein and TagRFP tagged AP2A1, both of which are components of the endocytic machinery, from the plasma membrane concurrently. Apart from all of the above, nanobodies are applied to protein purification as well. Anti-GFP nanobodies were coupled to N-hydroxysuccinimide-Sepharose and purified GFP proteins from root of Arabidopsis seedlings (Rossi et al. 2017).

\section{Targeted degradation}

Compared with gene-editing and RNA interference, direct manipulation of biomolecular pathways at the protein level is more effective for protein function studies. Immunomodulation of desired targets through intrabodies like nanobodies is emerging as an excellent tool. Nanobodies have been adopted to target POIs for specific degradation through ubiquitin-proteasome system (UPS). Degrons, protein domains that recruit the fusion protein to E3 ubiquitin ligase complex, are fused with nanobodies targeting POIs for their ubiquitylation and proteasome-mediated degradation (Ingram et al. 2018; Natsume and Kanemaki 2017). To achieve controlled and reversible degradation, various inducible degrons have been developed to degrade POIs in an auxin, light, or temperature-dependent manner (Natsume and Kanemaki 2017). deGradFP was developed to degrade GFP-tagged POIs specifically through the fusion of antiGFP nanobody with F-box domain, which is a component of the SKP1-CUL1-F-box (SCF) ubiquitin E3 ligases complex, and the POIs degradation is visible via fluorescence microscopy (Caussinus et al. 2011). An auxin-inducible degron (AID) has been introduced to update the system (Daniel et al. 2018). In plants, the deGradFP system was first applied to tobacco successfully (Baudisch et al. 2018). In Arabidopsis, in order to explore the role of WUS protein in maintaining the balance between self-renewal and differentiation in stem cells, Ma et al. (2019) applied anti-GFP nanobodies under the control of ethanol-inducible AlcR/AlcA system to degrade GFP tagged WUS protein in a switchable manner. The expression of WUS-linker-GFP was found to be substantially reduced in stem cells of the epidermis and subepidermis after $24 \mathrm{~h}$ induction of nanobody expression. Endogenous plant proteins can also be degraded in a controlled and reversible way using their specific nanobodies.

\section{Structure-based modulation and surveillance of protein function}

Since nanobodies are able to recognize cryptic epitopes formed by discontinuous amino acid segments, such as protein surface clefts, interfaces between domains, subunits, and interacted proteins, they can be used as tools to modulate enzyme activity, disturb proteins interaction as well as monitor or modulate protein conformations (Ingram et al. 2018; Pardon et al. 2014; Vincke and Muyldermans 2012). Research has shown nanobodies as competitive enzyme inhibitors of immunomodulation. Jobling et al. (2002) isolated nanobodies against potato starch branching enzyme A (SBE A) and correctly targeted them to subcellular organelles, thereby effectively reducing the enzyme activity of SBE A without affecting protein levels, which ultimately resulted in modified starch phenotype. The study by Vance et al. (2013) demonstrated that nanobodies were able to neutralize the plant-derived toxin. Ricin is a ribosome-inactivating protein (RIP) derived from the seed of castor bean plant, Ricinus communis, and is a lethal proteinaceous toxin classified as a Select Toxin by the Centers for Disease Control and Prevention due to its potential use as a bioterrorism agent. A collection of nanobodies specific for the ricin enzymatic subunit (RTA) and binding subunit (RTB) were isolated and characterized, among which six nanobodies had toxin-neutralizing activity. The authors linked the neutralizing RTA nanobodies with RTB nanobodies to create heterodimers, which were demonstrated to have higher affinities for ricin in vivo. Further, to probe into the mechanisms underlying toxin neutralization, the authors co-crystallized RTA with its specific nanobodies, and determined the crystal structures (Rudolph et al. 2014), and differential neutralizing activities of nanobody against RTB were also characterized (Herrera et al. 2014). Nanobodies have also been used as protein conformational biosensors. Nb80 was a conformation-specific nanobody that selectively recognized the active form of 
GPCR, and was used as an indicator of GPCR activation. In this research, in the absence of GPCR agonists, Nb80-GFP fusion protein localized to the cytoplasm in cells, whereas after agonist application, Nb80-GFP was recruited to the activated GPCR in the plasma membrane rapidly (Irannejad et al. 2013). However, nanobodies conformation monitoring has not been applied to plants yet. In structural biology, nanobodies have been used as excellent crystallization chaperones to stabilize proteins conformation. Enhanced disease susceptibility 1 (EDS1) functions as a central hub of TIR-type NLR regulated signaling through assembling with phytoalexin-deficient 4 (PAD4) or senescence associated gene 101 (SAG101) in plant innate immunity. Although the crystal structure of EDS1/SAG101 heterodimer has been solved, pure EDS1 is difficult in crystallization. Voss et al. (2019) produced EDS1-specific nanobodies as crystallization chaperones to solve the structure of EDS1 in solution, and demonstrated Arabidopsis EDS1 to be in monomeric protein state instead of forming homodimers when they were not associated with PAD4 or SAG101.

\section{Detection of plant toxins and pathogens}

Since nanobodies are robust to harsh conditions and are able to refold properly to recover their antigen affinity after denaturalization (Dumoulin et al. 2002), they are suitable capture agents for immunodetection. Nanobodies have been widely applied to the detection of plant-derived toxins or plant pathogens. Shia and Bailey (2013) adopted anti-ricin nanobodies in a label-free microring array detection platform to rapidly detect ricin with concentration as low as $300 \mathrm{pM}$. Moreover, they demonstrated that nanobodies were superior to conventional antibody with higher sensitivity and higher specificity in distinguishing ricin from the non-toxic ricin analogue RCA 120, which also existed in the castor bean plant. Besides, nanobodies have also been applied to detection of transgene expressed toxins in plants. Bacillus thuringiensis $(B t)$ derived Cry toxins are widely utilized as biological pesticides of insects in genetically modified (GM) crops. However, they have been identified to cause adverse effects on human health and the environment. Therefore, nanobodies were isolated for sensitive and specific detection of various types of Cry proteins in transgenic Bt plants based on phage-mediated ELISA (Li et al. 2014; Zhong et al. 2018) or immune-PCR methods (Liu et al. 2016). In addition to phytotoxins, nanobodies are also isolated for the early detection of plant pathogens, which helps plant disease prevention and control. Tulip virus $X(\mathrm{TuVX})$ is a filamentous, positivestrand RNA virus that is one of the abundant pathogens for tulip. Beekwilder et al. (2008) obtained several nanobodies through immunizing alpaca with TuVX and phage biopanning, and demonstrated that these nanobodies were able to distinguish TuVX-infected tulip leaves from uninfected leaves through ELISA. Additionally, the authors found that these nanobodies were specific for TuVX rather than other virus of the same family. In another research, nanobodies were isolated against Zucchini yellow mosaic virus (ZYMV) and could be used in virus detection with high specificity (Adel et al. 2018).

\section{Nanobody-mediated resistance against plant pathogens}

Compared with the wide therapeutic application of nanobodies in human diseases and pathogens, relevant applications in plants are limited until now. However, ectopic expression of antigen-specific nanobodies in plants is promising in establishing resistance against pathogens to reduce crop loss. Nanobody binding to the key proteins of pathogens are capable of preventing their cell attachment, invasion, reproduction, and movement, thus interrupting their life cycles. In addition, nanobodies can also directly neutralize the toxins secreted by pathogens and reduce the damage to plants. Boonrod et al. (2004) generated single-chain Fv fragments (scFvs) against a conserved domain in a plant viral RNAdependent RNA polymerase (RdRp) and conferred plants resistance to the virus through inhibiting virus replication. However, since the stability of scFvs relies on intrachain disulfide bonds, it is challenging to express scFvs in the cytoplasm, which is feasible for nanobodies because their stability is less dependent on the disulfide bond formation (Ingram et al. 2018). Broad bean mottle virus (BBMV) is a seed-born plant virus that infects Cicer arietinum, Pisum sativum, and Vicia faba, and causes great economic loss in the Mediterranean region. Ghannam et al. (2015) isolated eight BBMV-specific nanobodies with high affinity by phage display bio-panning from a library constructed from camel immunizations. The authors demonstrated that three of them successfully attenuated the BBMV spreading in vitro, and these nanobodies were capable of neutralizing BBMV when transiently expressed in Vicia faba. A recent excellent study involves Grapevine fanleaf virus (GFLV), which is a nematode-transmitted picorna-like plant virus affecting vineyards worldwide. Hemmer et al. (2018) isolated GFLVinhibiting nanobody, $\mathrm{Nb} 23$, and stably expressed it in Nicotiana benthamiana as well as in the grapevine rootstock, the natural host of the virus. In both cases, they observed strong and specific resistance against GFLV. To explore the mechanism of $\mathrm{Nb} 23$-mediated inhibition of the virus, the authors determined the structure of the GFLV-Nb23 complex by high-resolution single-particle cryo electron microscopy. The structure revealed that $\mathrm{Nb} 23$ bound to the GFLV capsid surface, which was a composite binding site involving three domains of one capsid protein (CP) monomer. Consistently, 
epitope mapping of the resistance-breaking GFLV variants showed that their CPs had C-terminal extensions, which sterically prevent the binding of Nb23 (Orlov et al. 2020). We look forward to more researches in this field, especially nanobody applications in resistance against plant pathogenic bacteria and fungi.

\section{Concluding remarks}

Nanobodies have excellent properties superior to traditional antibodies, including high stability with recoverable foldability, capability of recognizing cryptic epitopes and protein conformations, ease of genetic engineering, suitability of screening using synthetic libraries. These characteristics have made nanobodies powerful tools for various biomedical and biotechnological applications in plants and animals.

Plants are excellent host for the expression of therapeutic proteins, especially during the outbreak of a pandemic disease like the ongoing COVID-19. They can be scaledup easily and produce urgently needed antibodies, vaccines in a short time, which is challenging and costly for other systems (Capell et al. 2020). At present, only a small number of nanobodies are directed against plant proteins, thus limiting their applications. With nanobodies against plant endogenous proteins, it is possible to regulate and monitor the activity/conformation of plant proteases or receptor proteins, determine protein structures, regulate metabolic network to control plant metabolite production, develop nanobody-based plant biosensors, and control plants responses to environments. The use of nanobodies directed against key proteins of plant pathogens can interfere with their life cycles and enhance plant resistance specifically. Currently, such applications are mainly directed against plant viruses. Thus, more applications aiming at viruses, bacteria, and fungi are worth looking forward to.

Nanobodies are promising tools in agronomic applications of nanotechnology, which enables the controlled and targeted release of agrochemicals (fertilizers, insecticides, herbicides, etc.) (Wang et al. 2016a), and also the engineering of smart plant sensors (Giraldo et al. 2019). Nanobodies can be easily tailored for conjugating to nanoparticles and improve their active targeting. It is worth noting that the rigid cell wall outside the plant cell membrane, which is absent in animal cells, is a physical barrier for nanoparticles entry and apoplastic transport. However, since the size exclusion limits (SELs) of the cell wall are within 5-20 nm in diameter (Wang et al. 2016a), nanobodies in this size range are likely to be able to easily pass through the cell wall. And it is also reasonable to deduce the symplastic transport of nanobodies between the cytoplasm of adjacent cells facilitated by plasmodesmata with SEL of 3-50 nm (Sanzari et al. 2019; Wang et al. 2016a). Nevertheless, it is necessary to investigate the mechanisms of uptake and translocation of nanobodies in plants.

Additionally, since nanobodies are highly adaptable and easy to format into bivalent or multivalent antibodies, or fuse with other functional proteins to form versatile and multispecific modules, they provide plant biologists with powerful tools to achieve the above manipulations. Undoubtedly, further researches will expand the applications of nanobodies in plant in future years.

Author contributions WW formulated the outline of the review, drew figures, and wrote the manuscript. CJ and JY edited the manuscript. All authors contributed to the review.

Funding This work was supported by Zhuhai Talent Recruitment Grant for the Tumor Precision Diagnosis Innovation Team (Grant No. ZH0111-0405-180059-P-WC).

\section{Compliance with ethical standards}

Conflict of interest The authors declare no conflicts of interest.

\section{References}

Abe $\mathrm{M}$ et al (2014) A rice-based soluble form of a murine TNF-specific llama variable domain of heavy-chain antibody suppresses collagen-induced arthritis in mice. J Biotechnol 175:45-52. https ://doi.org/10.1016/j.jbiotec.2014.02.005

Adel MZ et al (2018) Cloning and characterisation of nanobodies against the coat protein of Zucchini yellow mosaic virus. Plant Prot Sci 54:215-221. https://doi.org/10.17221/158/2017-pps

Alvarez ML, Pinyerd HL, Topal E, Cardineau GA (2008) P19-dependent and P19-independent reversion of F1-V gene silencing in tomato. Plant Mol Biol 68:61-79. https://doi.org/10.1007/s1110 3-008-9352-2

Alvarez ML, Topal E, Martin F, Cardineau GA (2010) Higher accumulation of F1-V fusion recombinant protein in plants after induction of protein body formation. Plant Mol Biol 72:75-89. https://doi. org/10.1007/s11103-009-9552-4

Arbabi-Ghahroudi M, Tanha J, MacKenzie R (2005) Prokaryotic expression of antibodies. Cancer Metastasis Rev 24:501-519. https://doi.org/10.1007/s10555-005-6193-1

Barrera DJ et al (2015) Algal chloroplast produced camelid $\mathrm{V}_{\mathrm{H}} \mathrm{H}$ antitoxins are capable of neutralizing botulinum neurotoxin. Plant Biotechnol J 13:117-124. https://doi.org/10.1111/pbi.12244

Baudisch B, Pfort I, Sorge E, Conrad U (2018) Nanobody-directed specific degradation of proteins by the 26S-proteasome in plants. Front Plant Sci 9:130. https://doi.org/10.3389/fpls.2018.00130

Beekwilder J, van Houwelingen A, van Beckhoven J, Speksnijder A (2008) Stable recombinant alpaca antibodies for detection of Tulip virus X. Eur J Plant Pathol 121:477-485. https://doi.org/10.1007/ s10658-007-9265-y

Boonrod K et al (2004) Single-chain antibodies against a plant viral RNA-dependent RNA polymerase confer virus resistance. Nat Biotechnol 22:856-862. https://doi.org/10.1038/nbt983

Boothe J et al (2010) Seed-based expression systems for plant molecular farming. Plant Biotechnol J 8:588-606. https://doi.org/10. 1111/j.1467-7652.2010.00511.x 
Borg S, Popp F, Hofmann J, Leonhardt H, Rothbauer U, Schuler D (2015) An intracellular nanotrap redirects proteins and organelles in live bacteria. mBio. https://doi.org/10.1128/ mBio.02117-14

Capell T, Twyman RM, Armario-Najera V, Ma JK, Schillberg S, Christou P (2020) Potential applications of plant biotechnology against SARS-CoV-2. Trends Plant Sci. https://doi.org/10.1016/j.tplan ts.2020.04.009

Caussinus E, Kanca O, Affolter M (2011) Fluorescent fusion protein knockout mediated by anti-GFP nanobody. Nat Struct Mol Biol 19:117-121. https://doi.org/10.1038/nsmb.2180

Chakravarty R, Goel S, Cai W (2014) Nanobody: the "magic bullet" for molecular imaging? Theranostics 4:386-398. https://doi. org/10.7150/thno.8006

Chen M, Liu X, Wang Z, Song J, Qi Q, Wang PG (2005) Modification of plant $\mathrm{N}$-glycans processing: the future of producing therapeutic protein by transgenic plants. Med Res Rev 25:343-360. https:// doi.org/10.1002/med.20022

Conrad U et al (2011) ELPylated anti-human TNF therapeutic single-domain antibodies for prevention of lethal septic shock. Plant Biotechnol J 9:22-31. https://doi.org/10.111 1/j.1467-7652.2010.00523.x

Daniel K, Icha J, Horenburg C, Müller D, Norden C, Mansfeld J (2018) Conditional control of fluorescent protein degradation by an auxin-dependent nanobody. Nat Commun. https://doi. org/10.1038/s41467-018-05855-5

Daniell H, Singh ND, Mason H, Streatfield SJ (2009) Plant-made vaccine antigens and biopharmaceuticals. Trends Plant Sci 14:669679. https://doi.org/10.1016/j.tplants.2009.09.009

De Buck S et al (2013) Fusion of an Fc chain to a VHH boosts the accumulation levels in Arabidopsis seeds. Plant Biotechnol J 11:1006-1016. https://doi.org/10.1111/pbi.12094

De Genst E et al (2006) Molecular basis for the preferential cleft recognition by dromedary heavy-chain antibodies. Proc Natl Acad Sci USA 103:4586-4591. https://doi.org/10.1073/pnas.0505379103

De Greve H, Virdi V, Bakshi S, Depicker A (2020) Simplified monomeric VHH-Fc antibodies provide new opportunities for passive immunization. Curr Opin Biotechnol 61:96-101. https://doi. org/10.1016/j.copbio.2019.11.006

De Vos J, Devoogdt N, Lahoutte T, Muyldermans S (2013) Camelid single-domain antibody-fragment engineering for (pre)clinical in vivo molecular imaging applications: adjusting the bullet to its target. Expert Opin Biol Ther 13:1149-1160. https://doi. org/10.1517/14712598.2013.800478

Desmyter A et al (1996) Crystal structure of a camel single-domain VH antibody fragment in complex with lysozyme. Nat Struct Biol 3:803-811. https://doi.org/10.1038/nsb0996-803

Dumoulin M et al (2002) Single-domain antibody fragments with high conformational stability. Protein Sci 11:500-515. https:// doi.org/10.1110/ps.34602

Fruholz S, Fassler F, Kolukisaoglu U, Pimpl P (2018) Nanobody-triggered lockdown of VSRs reveals ligand reloading in the Golgi. Nat Commun 9:643. https://doi.org/10.1038/s41467-018-02909-6

Ghannam A, Kumari S, Muyldermans S, Abbady AQ (2015) Camelid nanobodies with high affinity for broad bean mottle virus: a possible promising tool to immunomodulate plant resistance against viruses. Plant Mol Biol 87:355-369. https://doi.org/10.1007/ s11103-015-0282-5

Giersberg M, Floss DM, Kipriyanov S, Conrad U, Scheller J (2010) Covalent dimerization of camelidae anti-human TNF-alpha single domain antibodies by the constant kappa light chain domain improves neutralizing activity. Biotechnol Bioeng 106:161-166. https://doi.org/10.1002/bit.22653

Giraldo JP, Wu H, Newkirk GM, Kruss S (2019) Nanobiotechnology approaches for engineering smart plant sensors. Nat Nanotechnol 14:541-553. https://doi.org/10.1038/s41565-019-0470-6
Harmsen MM, De Haard HJ (2007) Properties, production, and applications of camelid single-domain antibody fragments. Appl Microbiol Biotechnol 77:13-22. https://doi.org/10.1007/s0025 3-007-1142-2

Harmsen MM, van Solt CB, Hoogendoorn A, van Zijderveld FG, Niewold TA, van der Meulen J (2005) Escherichia coli F4 fimbriae specific llama single-domain antibody fragments effectively inhibit bacterial adhesion in vitro but poorly protect against diarrhoea. Vet Microbiol 111:89-98. https://doi.org/10.1016/j.vetmi c.2005.09.005

Hemmer C et al (2018) Nanobody-mediated resistance to Grapevine fanleaf virus in plants. Plant Biotechnol J 16:660-671. https://doi. org/10.1111/pbi.12819

Herrera C, Vance DJ, Eisele LE, Shoemaker CB, Mantis NJ (2014) Differential neutralizing activities of a single domain camelid antibody $\left(\mathrm{V}_{\mathrm{H}} \mathrm{H}\right)$ specific for ricin toxin's binding subunit (RTB). PLoS ONE 9:e99788. https://doi.org/10.1371/journal.pone.0099788

Hussack G, Hirama T, Ding W, Mackenzie R, Tanha J (2011) Engineered single-domain antibodies with high protease resistance and thermal stability. PLoS ONE 6:e28218. https://doi.org/10.1371/ journal.pone.0028218

Hutt M, Farber-Schwarz A, Unverdorben F, Richter F, Kontermann RE (2012) Plasma half-life extension of small recombinant antibodies by fusion to immunoglobulin-binding domains. J Biol Chem 287:4462-4469. https://doi.org/10.1074/jbc.M111.311522

Ingram JR, Schmidt FI, Ploegh HL (2018) Exploiting nanobodies' singular traits. Annu Rev Immunol 36:695-715. https://doi. org/10.1146/annurev-immunol-042617-053327

Irannejad R et al (2013) Conformational biosensors reveal GPCR signalling from endosomes. Nature 495:534-538. https://doi. org/10.1038/nature 12000

Ismaili A, Jalali-Javaran M, Rasaee MJ, Rahbarizadeh F, ForouzandehMoghadam M, Memari HR (2007) Production and characterization of anti-(mucin MUC1) single-domain antibody in tobacco (Nicotiana tabacum cultivar Xanthi). Biotechnol Appl Biochem 47:11-19. https://doi.org/10.1042/BA20060071

Jin S, Daniell H (2015) The engineered chloroplast genome just got smarter. Trends Plant Sci 20:622-640. https://doi.org/10.1016/j. tplants.2015.07.004

Jobling SA, Jarman C, Teh M-M, Holmberg N, Blake C, Verhoeyen ME (2002) Immunomodulation of enzyme function in plants by single-domain antibody fragments. Nat Biotechnol 21:77-80. https://doi.org/10.1038/nbt772

Jovcevska I, Muyldermans S (2020) The therapeutic potential of nanobodies. BioDrugs 34:11-26. https://doi.org/10.1007/s40259-01900392-z

Kunzl F, Fruholz S, Fassler F, Li B, Pimpl P (2016) Receptormediated sorting of soluble vacuolar proteins ends at the transGolgi network/early endosome. Nat Plants 2:16017. https://doi. org/10.1038/nplants.2016.17

Lentz EM, Garaicoechea L, Alfano EF, Parreno V, Wigdorovitz A, Bravo-Almonacid FF (2012) Translational fusion and redirection to thylakoid lumen as strategies to improve the accumulation of a camelid antibody fragment in transplastomic tobacco. Planta 236:703-714. https://doi.org/10.1007/s00425-012-1642-x

Li M, Zhu M, Zhang C, Liu X, Wan Y (2014) Uniform orientation of biotinylated nanobody as an affinity binder for detection of Bacillus thuringiensis (Bt) Cry1Ac toxin. Toxins (Basel) 6:3208-3222. https://doi.org/10.3390/toxins6123208

Liu W, Song H, Chen Q, Yu J, Xian M, Nian R, Feng D (2018) Recent advances in the selection and identification of antigen-specific nanobodies. Mol Immunol 96:37-47. https://doi.org/10.1016/j. molimm.2018.02.012

Liu Y, Huang H (2018) Expression of single-domain antibody in different systems. Appl Microbiol Biotechnol 102:539-551. https:// doi.org/10.1007/s00253-017-8644-3 
Liu Y, Jiang D, Lu X, Wang W, Xu Y, He Q (2016) Phage-mediated immuno-PCR for ultrasensitive detection of Cry1Ac protein based on nanobody. J Agric Food Chem 64:7882-7889. https:// doi.org/10.1021/acs.jafc.6b02978

Ma Y et al (2019) WUSCHEL acts as an auxin response rheostat to maintain apical stem cells in Arabidopsis. Nat Commun 10:5093. https://doi.org/10.1038/s41467-019-13074-9

Manta B, Boyd D, Berkmen M (2019) Disulfide bond formation in the periplasm of Escherichia coli. EcoSal Plus. https://doi. org/10.1128/ecosalplus.ESP-0012-2018

Mirzaee M, Jalali-Javaran M, Moieni A, Zeinali S, Behdani M (2018) Expression of VGRNb-PE immunotoxin in transplastomic lettuce (Lactuca sativa L.). Plant Mol Biol 97:103-112. https://doi. org/10.1007/s11103-018-0726-9

Modarresi M, Javaran MJ, Shams-Bakhsh M, Zeinali S, Behdani M, Mirzaee M (2018) Transient expression of anti-VEFGR2 nanobody in Nicotiana tabacum and N. benthamiana. 3 Biotech 8:484. https://doi.org/10.1007/s13205-018-1500-z

Moutel S et al (2016) NaLi-H1: a universal synthetic library of humanized nanobodies providing highly functional antibodies and intrabodies. Elife. https://doi.org/10.7554/eLife.16228

Muyldermans S (2013) Nanobodies: natural single-domain antibodies. Annu Rev Biochem 82:775-797. https://doi.org/10.1146/annurevbiochem-063011-092449

Muyldermans S et al (2009) Camelid immunoglobulins and nanobody technology. Vet Immunol Immunopathol 128:178-183. https:// doi.org/10.1016/j.vetimm.2008.10.299

Natsume T, Kanemaki MT (2017) Conditional degrons for controlling protein expression at the protein level. Annu Rev Genet 51:83102. https://doi.org/10.1146/annurev-genet-120116-024656

Orlov I et al (2020) Structural basis of nanobody recognition of grapevine fanleaf virus and of virus resistance loss. Proc Natl Acad Sci USA 117:10848-10855. https://doi.org/10.1073/pnas.1913681117

Orzaez D, Granell A, Blazquez MA (2009) Manufacturing antibodies in the plant cell. Biotechnol J 4:1712-1724. https://doi. org/10.1002/biot.200900223

Palaci J, Virdi V, Depicker A (2019) Transformation strategies for stable expression of complex hetero-multimeric proteins like secretory immunoglobulin A in plants. Plant Biotechnol J 17:17601769. https://doi.org/10.1111/pbi.13098

Pardon E et al (2014) A general protocol for the generation of Nanobodies for structural biology. Nat Protoc 9:674-693. https://doi. org/10.1038/nprot.2014.039

Park SR et al (2020) Expression and in vitro function of anti-breast cancer llama-based single domain antibody VHH expressed in tobacco plants. Int J Mol Sci. https://doi.org/10.3390/ijms210413 54

Rajabi-Memari H, Jalali-Javaran M, Rasaee MJ, Rahbarizadeh F, Forouzandeh-Moghadam M, Esmaili A (2006) Expression and characterization of a recombinant single-domain monoclonal antibody against MUC1 mucin in tobacco plants. Hybridoma (Larchmt) 25:209-215. https://doi.org/10.1089/hyb.2006.25.209

Richard G, Meyers AJ, McLean MD, Arbabi-Ghahroudi M, MacKenzie R, Hall JC (2013) In vivo neutralization of $\alpha$-cobratoxin with high-affinity llama single-domain antibodies $\left(\mathrm{V}_{\mathrm{H}} \mathrm{Hs}\right)$ and a $\mathrm{V}_{\mathrm{H}} \mathrm{H}-\mathrm{Fc}$ antibody. PLoS ONE 8:e69495. https://doi.org/10.1371/ journal.pone.0069495

Rocchetti A, Hawes C, Kriechbaumer V (2014) Fluorescent labelling of the actin cytoskeleton in plants using a cameloid antibody. Plant Methods 10:12. https://doi.org/10.1186/1746-4811-10-12

Roche AM, Richard AL, Rahkola JT, Janoff EN, Weiser JN (2015) Antibody blocks acquisition of bacterial colonization through agglutination. Mucosal Immunol 8:176-185. https://doi. org/10.1038/mi.2014.55
Rossi MT, Kalde M, Srisakvarakul C, Kruger NJ, Ratcliffe RG (2017) Cell-type specific metabolic flux analysis: a challenge for metabolic phenotyping and a potential solution in plants. Metabolites. https://doi.org/10.3390/metabo7040059

Rothbauer U, Zolghadr K, Muyldermans S, Schepers A, Cardoso MC, Leonhardt H (2008) A versatile nanotrap for biochemical and functional studies with fluorescent fusion proteins. Mol Cell Proteomics 7:282-289. https://doi.org/10.1074/mcp.M7003 42-MCP200

Rothbauer U et al (2006) Targeting and tracing antigens in live cells with fluorescent nanobodies. Nat Methods 3:887-889. https://doi. org/10.1038/nmeth953

Rudolph MJ et al (2014) Crystal structures of ricin toxin's enzymatic subunit (RTA) in complex with neutralizing and non-neutralizing single-chain antibodies. J Mol Biol 426:3057-3068. https://doi. org/10.1016/j.jmb.2014.05.026

Saberianfar R, Chin-Fatt A, Scott A, Henry KA, Topp E, Menassa R (2019) Plant-produced chimeric $\mathrm{V}_{\mathrm{H}} \mathrm{H}$-sIgA against enterohemorrhagic $E$. coli intimin shows cross-serotype inhibition of bacterial adhesion to epithelial cells. Front Plant Sci 10:270. https://doi. org/10.3389/fpls.2019.00270

Salvador JP, Vilaplana L, Marco MP (2019) Nanobody: outstanding features for diagnostic and therapeutic applications. Anal Bioanal Chem 411:1703-1713. https://doi.org/10.1007/s00216-019-01633 $-4$

Sanzari I, Leone A, Ambrosone A (2019) Nanotechnology in plant science: to make a long story short. Front Bioeng Biotechnol 7:120. https://doi.org/10.3389/fbioe.2019.00120

Schornack S, Fuchs R, Huitema E, Rothbauer U, Lipka V, Kamoun S (2009) Protein mislocalization in plant cells using a GFP-binding chromobody. Plant J 60:744-754. https://doi.org/10.1111/j.1365313X.2009.03982.X

Schumacher D, Helma J, Schneider AFL, Leonhardt H, Hackenberger CPR (2018) Nanobodies: chemical functionalization strategies and intracellular applications. Angew Chem Int Ed Engl 57:2314 2333. https://doi.org/10.1002/anie.201708459

Shia WW, Bailey RC (2013) Single domain antibodies for the detection of ricin using silicon photonic microring resonator arrays. Anal Chem 85:805-810. https://doi.org/10.1021/ac3030416

Soleimanizadeh M, Bagheri A, Jalali Javaran M, Seifi A, Behdani M, Kazemi-Lomedasht F (2018) Enhanced expression and purification of anti-VEGF nanobody in cucurbit plants. J Plant Biochem Biotechnol 28:263-270. https://doi.org/10.1007/s1356 2-018-0471-9

Strasser R, Altmann F, Steinkellner H (2014) Controlled glycosylation of plant-produced recombinant proteins. Curr Opin Biotechnol 30:95-100. https://doi.org/10.1016/j.copbio.2014.06.008

Streatfield SJ (2007) Approaches to achieve high-level heterologous protein production in plants. Plant Biotechnol J 5:2-15. https:// doi.org/10.1111/j.1467-7652.2006.00216.x

Teh YH, Kavanagh TA (2010) High-level expression of Camelid nanobodies in Nicotiana benthamiana. Transgenic Res 19:575-586. https://doi.org/10.1007/s11248-009-9338-0

Topp E et al (2016) The case for plant-made veterinary immunotherapeutics. Biotechnol Adv 34:597-604. https://doi.org/10.1016/j. biotechadv.2016.02.007

Tschofen M, Knopp D, Hood E, Stöger E (2016) Plant molecular farming: much more than medicines. Annu Rev Anal Chem 9:271294. https://doi.org/10.1146/annurev-anchem-071015-041706

Van Droogenbroeck B et al (2007) Aberrant localization and underglycosylation of highly accumulating single-chain Fv-Fc antibodies in transgenic Arabidopsis seeds. Proc Natl Acad Sci USA 104:1430-1435. https://doi.org/10.1073/pnas.0609997104

Vance DJ, Tremblay JM, Mantis NJ, Shoemaker CB (2013) Stepwise engineering of heterodimeric single domain camelid $\mathrm{V}_{\mathrm{H}} \mathrm{H}$ 
antibodies that passively protect mice from ricin toxin. J Biol Chem 288:36538-36547. https://doi.org/10.1074/jbc.M113.51920 7

Vanmarsenille C et al (2018) In planta expression of nanobody-based designer chicken antibodies targeting Campylobacter. PLoS ONE 13:e0204222. https://doi.org/10.1371/journal.pone.0204222

Vezina LP et al (2009) Transient co-expression for fast and highyield production of antibodies with human-like $\mathrm{N}$-glycans in plants. Plant Biotechnol J 7:442-455. https://doi.org/10.111 1/j.1467-7652.2009.00414.x

Vincke C, Muyldermans S (2012) Introduction to heavy chain antibodies and derived Nanobodies. Methods Mol Biol 911:15-26. https ://doi.org/10.1007/978-1-61779-968-6_2

Virdi V et al (2013) Orally fed seeds producing designer IgAs protect weaned piglets against enterotoxigenic Escherichia coli infection. Proc Natl Acad Sci 110:11809-11814. https://doi.org/10.1073/ pnas. 1301975110

Virdi V, Juarez P, Depicker A (2015) Plant expression systems for early stage discovery and development of lead therapeutic antibodies. Hum Antib 23:37-43. https://doi.org/10.3233/HAB-150285

Virdi V et al (2019) Yeast-secreted, dried and food-admixed monomeric IgA prevents gastrointestinal infection in a piglet model. Nat Biotechnol 37:527-530. https://doi.org/10.1038/s4158 7-019-0070-x

Voss M, Toelzer C, Bhandari DD, Parker JE, Niefind K (2019) Arabidopsis immunity regulator EDS1 in a PAD4/SAG101-unbound form is a monomer with an inherently inactive conformation. $\mathrm{J}$ Struct Biol 208:107390. https://doi.org/10.1016/j.jsb.2019.09.007

Wang P, Lombi E, Zhao FJ, Kopittke PM (2016a) Nanotechnology: a new opportunity in plant sciences. Trends Plant Sci 21:699-712. https://doi.org/10.1016/j.tplants.2016.04.005

Wang Y et al (2016b) Nanobody-derived nanobiotechnology tool kits for diverse biomedical and biotechnology applications. Int J Nanomed 11:3287-3303. https://doi.org/10.2147/IJN.S107194

Whaley KJ, Hiatt A, Zeitlin L (2011) Emerging antibody products and Nicotiana manufacturing. Hum Vaccin 7:349-356. https:// doi.org/10.4161/hv.7.3.14266
Winichayakul S, Pernthaner A, Scott R, Vlaming R, Roberts N (2009) Head-to-tail fusions of camelid antibodies can be expressed in planta and bind in rumen fluid. Biotechnol Appl Biochem. https ://doi.org/10.1042/ba20080076

Winkler J, De Meyer A, Mylle E, Grones P, Van Damme D (2020) Nanobody-dependent delocalization of endocytic machinery in Arabidopsis root cells dampens their internalization capacity. BioRxiv. https://doi.org/10.1101/2020.02.27.968446

Xu J, Dolan MC, Medrano G, Cramer CL, Weathers PJ (2012) Green factory: plants as bioproduction platforms for recombinant proteins. Biotechnol Adv 30:1171-1184. https://doi.org/10.1016/j. biotechadv.2011.08.020

Yan J, Li G, Hu Y, Ou W, Wan Y (2014) Construction of a synthetic phage-displayed Nanobody library with CDR3 regions randomized by trinucleotide cassettes for diagnostic applications. J Transl Med 12:343. https://doi.org/10.1186/s12967-014-0343-6

Zhong W, Li G, Yu X, Zhu M, Gong L, Wan Y (2018) Sensitive detection of Bacillus thuringiensis Cry1B toxin based on camel single-domain antibodies. Microbiologyopen 7:e00581. https://doi. org $/ 10.1002 / \mathrm{mbo} 3.581$

Zimmermann I et al (2020) Generation of synthetic nanobodies against delicate proteins. Nat Protoc 15:1707-1741. https://doi. org/10.1038/s41596-020-0304-x

Zimmermann J et al (2009) Antibody expressing pea seeds as fodder for prevention of gastrointestinal parasitic infections in chickens. BMC Biotechnol 9:79. https://doi.org/10.1186/1472-6750-9-79

Publisher's Note Springer Nature remains neutral with regard to jurisdictional claims in published maps and institutional affiliations. 